\title{
Investigation of Irisin Level in Brucella Patients
}

\author{
Sermin Algul ${ }^{\star}$, Feray Ferda Senol ${ }^{2}$, Seda Ugras ${ }^{3}$, Fatih Esmer ${ }^{4}$, Mehmet Kara $^{1}$, Pınar Ekici ${ }^{5}$, \\ Oguz Ozcelik ${ }^{6}$
}

${ }^{1}$ Van Yuzuncu Yil University, Faculty of Medicine, Department of Physiology, Van, Turkey

${ }^{2}$ Elazig Fethi Sekin City Hospital, Department of Microbiology and Clinical Microbiology, Elarig, Turkey

${ }^{3}$ Bozok University, Faculty of Medicine, Department of Physiology, Yozgat, Turkey

${ }^{4}$ Sanluurfa Mehmet Akif Inan Education and Research Hospital, Department of Infectious Diseases and Clinical

Microbiology, Sanliurfa, Turkey

${ }^{5}$ Van Yu₹uncu Yil University, Faculty of Health Sciences, Department of Midwifery, Van, Turkey

${ }^{6}$ Kastamonu University, Faculty of Medicine, Department of Physiology, Kastamonu, Turkey

\begin{abstract}
This study aimed to determine whether the changes in the metabolism of Brucella patients affect the irisin hormone. Also, it is aimed to investigate the relationships between other laboratory findings used in the diagnosis of brucellosis patients and the irisin hormone.

160 persons ( 80 brucellosis, 80 controls) were included in this study. Blood was drawn from the people participating in the study following their overnight fast. Irisin level was measured by ELISA method, CRP and sedimentation levels were measured using autoanalysers and commercial kits.

It was determined that the irisin level of Brucella patients was statistically significantly higher than the control group ( $p<0.05)$. It was determined that there is a negative correlation between irisin level and CRP and sedimentation level in Brucella patients.

In Brucella patients, the higher irisin level than the control group showed a negative correlation with CRP and sedimentation levels, which are infection diagnosis criteria; It suggests that the level of irisin can be considered as a supportive diagnostic marker in these patients. Further studies are needed to evaluate irisin hormone as a supportive diagnostic criterion.
\end{abstract}

Key Words: Brucella, irisin, infection, C-reactive protein, sedimentation

\section{Introduction}

Brucella bacteria are intracellular, immobile, facultative anaerobe gram negative bacteria. Meat of animals such as sheep, goats, cattle, buffalo and pigs, body fluids such as milk and urine, dairy products prepared with infected milk, are an infectious agent of zooanthroponosis (transmitted from animals to humans) that can be transmitted to humans through pregnancy materials of infected animals (1).

The clinical signs of this inflammation are fever, arthritis, osteomyelitis, endocarditis, meningitis, pleocytosis, cellular infiltration of the joints, nephritis, hepatic granuloma (2). Brucella is an infectious agent that causes significant morbidity and mortality in developing countries. Brucella is very common in the Mediterranean Basin and the Arabian Peninsula, India, Mexico and Central and South America $(2,3)$. Turkey also has a high incidence of brucellosis and especially the Middle East and Southeast Anatolia regions are seen as much more common $(4,5)$. Brucella can mimic a variety of multi-system diseases with extensive clinical polymorphism and can often cause misdiagnosis and delay in treatment $(4,6)$. It can progress clinically as subclinical, acute, subacute and chronic infection $(4,6-8)$. Definitive diagnosis of brucellosis is made by determining the agent from blood and bone marrow samples. Detection of the agent may vary depending on the duration of the disease, the type of the agent, the method used, the amount in the circulation, the length of the incubation period and whether the patient has received antibiotic treatment before. For these reasons, serological tests are considered among the methods that support the preliminary diagnosis, have high sensitivity and specificity, are easy, cheap and give faster results than other methods, although they do not make a definitive diagnosis (4).

Recent studies have revealed the close relationship between the immune and endocrine systems. There are important study results that support bidirectional communication between neuroendocrine and immune system (9). First, hormone receptors were found in immune cells, and receptors for immune products were found in neural and endocrine cells (10).

\footnotetext{
*Corresponding Author: Assoc. Prof. Dr. Sermin Algul, Van Yuzuncu Yil University, Faculty of Medicine, Department of Physiology E-mail: serminalgul@hotmail.com, serminalgul@yyu.edu.tr, Phone: +90 (432) $2251701 / 25209$

ORCID ID: Sermin Algul: 0000-0003-2489-3619, Feray Ferda Senol: 0000-0003-4705-5757, Seda Ugras: 0000-0002-5768-7611, Fatih Esmer: 0000-0002-8512-5894, Mehmet Kara: 0000-0002-8864-9706, Pinar Ekici: 0000-0003-2719-5250, Oguz Ozcelik: 0000-0002-23919883
} 
Researchers suggest that the hormone irisin could be a promising agent for the effective treatment of many metabolic diseases such as obesity, diabetes, cardiovascular, thyroid hormone disorder, and others $(11,12)$. Irisin, a newly discovered hormone, is an exercise-induced peptide secreted from myocytes and adipocytes $(13,14$ 15). Exercise has significant effects on circulation irisin levels in trained and untrained subjects $(16,17)$. It has been suggested that the irisin also regulates various physiological and metabolic processes. Studies revealing the relation of the irisin to energy metabolic processes are available in the literature (11) however, there are no scientific studies revealing the relationship between irisin and infectious diseases.

Laboratory findings such as leukocytosis/leukopenia, relative lymphocytopenia, anemia, thrombocytopenia, high $C$ reactive protein and high erythrocyte sedimentation are used for diagnosis in patients with Brucella. However, these findings do not directly aid diagnosis (18). On the contrary, other markers such as mean platelet volume (MPV), neutrophil/lymphocyte ratio (NLR), and platelet/lymphocyte ratio are helpful criteria in the diagnosis of brucellosis (19-23).

It has been reported in the literature for many years that the neutrophil count can be used in the differential diagnosis of infectious diseases (24). It has also recently been reported that neutrophils may be another major source of irisin, an exercise-induced myocyte that spreads within the vascular structures (25).

It has also recently been reported that neutrophils may be another major source of irisin, an exerciseinduced myocyte that spreads within the vascular structures. Therefore, it may increase the irisin of the increased neutrophil count. It is suggested that measurement of irisin can be an additional supportive criterion that can be used in the differential diagnosis of infectious diseases such as brucellosis.

The aim of this study is to investigate whether the changes in the metabolism of Brucella patients, which are common in certain regions of our country, have effects on irisin, a metabolism hormone. In addition, it is aimed to determine the relationship between the other laboratory findings used in the diagnosis of brucellosis patients and the irisin hormone.

\section{Material and Method}

Eighty brucellosis patients ( $n=40$ females, $n=40$ males) and 80 healthy individuals without any infection ( $\mathrm{n}=40$ females, $\mathrm{n}=40$ males) participated in this study. The age, height and weight was $37.1 \pm$
14 years, $167 \pm 5 \mathrm{~cm}, 67.7 \pm 5.1 \mathrm{~kg}$ for Brucella patients, and $39 \pm 16$ years, $168 \pm 4 \mathrm{~cm}$, and $69.7 \pm$ $7.8 \mathrm{~kg}$ for the control group, respectively.

Ethical approval was obtained from Firat University Non-Invasive Local Ethics Committee before starting the study. This study protocol was conducted according to the Declaration of Helsinki.

Before starting the study, each participant underwent a medical examination including cardiovascular risk assessment (electrocardiography, medical history, physical examination, and hormone analysis to rule out abnormalities). It is known that different diseases such as gastrointestinal or nutritional disorders, metabolic diseases can significantly affect the level of irisin (11). Therefore, persons with any disease including respiratory, endocrinology, cardiac, metabolic or renal diseases and those with viral hepatitis were not included in the study. Additionally, people who took alcohol, cigarettes, vitamin supplements, any hormone or drug therapy, and people using substances were excluded from the study. People with a body mass index over 30, pregnant or receiving cancer treatment were not included in the study.

Specific diagnostic methods in Brucella: Showing culture and bacteria in direct method, showing antibodies; Standard tube agglutination, Rose Bengal slide test, Coombs test, Spot dipstick test, IFA test, ELISA, PCR. Rose Bengal, Standard tube agglutination, spot dipstick test, spot tests are fast, cheap and have high specificity in acute infections. ELISA and PCR methods are expensive and time consuming methods. In this study, Rose-Bengal lam agglutination test and standard tube agglutination test (STA) were performed in all patients with clinically suspected brucellosis. The STA test was performed by diluting the patient serum up to a titer of 1: 1280 and agglutinations with titers of 1: 160 and above were considered positive for brucellosis.

The control group consisted of healthy individuals who did not have brucellosis or any febrile infectious disease in the last 6 months. The control group was composed of people who did not take any vitamins or antioxidant supplements such as vitamin E and C.

Hormone Analysis: Patients were warned about not eating or drinking, exercising, and smoking before blood was drawn. All blood samples were taken between 08:00 and 09:00 in the morning following an overnight fast. Blood samples were centrifuged at +4 ${ }^{\circ} \mathrm{C} 4500 \mathrm{rpm}$ for 5 minutes and their serum was obtained. Serums obtained later were stored at $-80^{\circ} \mathrm{C}$ until analyzed. The level of irisin was measured by 


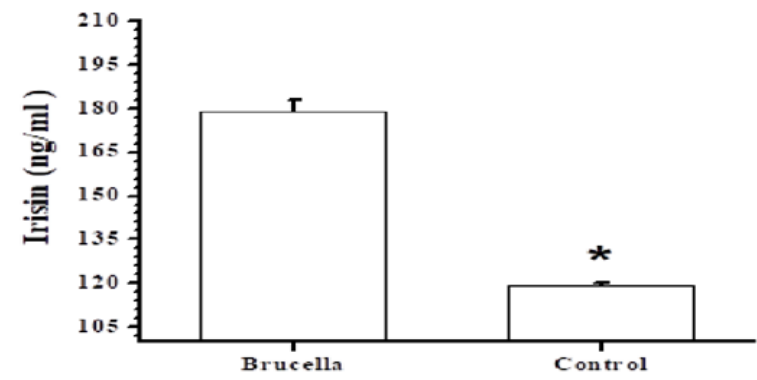

Fig. 1. Irisin levels response to the control, brucella patient's groups. Values are presented as mean \pm SE *significant differences compared to the control levels

ELISA method using a commercial kit with a sensitivity of $0.78 \mathrm{ng} / \mathrm{mL}$ (Phoenix Pharmaceuticals Inc, Burlingame, California, USA). Intra-inter assay coefficients are $5.61 \%$ and $14.56 \%$. All analyzes were carried out under the same experimental conditions with double blind. Serum CRP and sedimentation levels (Abbott Arcitech 8200) were measured in a Hemogram (Beckman Coulter) auto-analyzer using commercial kits.

Statistical Analysis: Values are expressed as mean \pm standard error (SE). The Kolmogorov-Smirnov Z test was performed and it was determined that the data showed normal distribution. Therefore, unpaired $t$ test was used to determine the difference between irisin levels in brucella and control groups. Pearson correlation coefficient was used to determine the correlation between irisin level and hematological parameters (CRP and sedimentation). $\mathrm{p}<0.05$ was considered statistically significant.

\section{Results}

When irisin levels of brucella and control groups were compared, it was determined that the irisin level in the brucella group (179.1 $\pm 3.6 \mathrm{ng} / \mathrm{ml})$ was statistically significantly higher than the irisin level $(119.0 \pm 1.2 \mathrm{ng} / \mathrm{ml})$ of the control group $(\mathrm{p}<0.05)$ (Figure).

When irisin level $(179.1 \pm 3.6 \mathrm{ng} / \mathrm{ml})$ and CRP level $(7.72 \pm 1.19 \mathrm{mg} / \mathrm{L})$ of Brucella patients were compared, it was determined that there was a statistically significant negative correlation between irisin and CRP levels $(\mathrm{p}<0.05, \mathrm{r}=-0.28)$.

When the irisin level $(179.1 \pm 3.6 \mathrm{ng} / \mathrm{ml})$ and sedimentation level $(15.52 \pm 1.95 \mathrm{~mm} / \mathrm{h})$ of Brucella patients were compared, it was determined that there was a statistically significant negative correlation between irisin and sedimentation levels $(\mathrm{p}<0.05, \mathrm{r}=$ $-0.37)$.

\section{Discussion}

The irisin is an exercise-induced hormone that increases fat burning by affecting human metabolism (13). In some diseases such as obesity and type 2 diabetes, the pathogenesis or relationship of the disease has been reported in the literature by affecting the level of irisin (11).

However, there are no studies investigating the level of irisin in infectious diseases. This is the first study investigating the level of the hormone irisin, a metabolism hormone, in patients with Brucella. In the literature, there are very few studies in which endocrine system hormones have been studied in brucellosis patients. However, studies reporting a close relationship between the endocrine system and the immune system are available in the literature (9, 10). In this study, the higher irisin level in brucellosis patients than the control group suggests that irisin hormone, which is an energy-metabolism hormone, may be a parameter that should be taken into account in patients with infection. Until now, blood irisin level has been studied in various diseases in the literature. Since it is known to be associated with muscle mass or insulin resistance, it has led to the investigation of its potential cardiovascular effect in various studies. Irisin level has been studied in chronic renal failure, some cancers, metabolic diseases, non-alcoholic liver disease and osteoporosis, but in these studies irisin was evaluated as a hormone rather than a biochemical marker (26). In this study, whether irisin level could be a biochemical marker in patients with brucellosis was investigated.

In a study conducted in the literature, irisin level was measured in rats with acute myocardial infarction. They determined that the level of the irisin decreased while on the contrary, the levels of CK and CK-MB increased (27). Therefore, they suggested that low irisin level in the early period could also be a biomarker to support the diagnosis of acute myocardial infarction. In this study, it was determined that there is a negative correlation between serum irisin level and CRP and sedimentation levels, which is an indicator of infection, in patients with brucellosis. Therefore, it is thought that serum irisin level in brucellosis patients can be considered as a supportive diagnostic marker. Additional supportive studies are needed for this.

Acknowledgement: This study was supported by Van Yüzüncü Y1l University Scientific Research Project Office (Project number: TSA-2018-6413). 


\section{References}

1. Baysal B. Brucella, (eds: Usyacelebi S.) Temel ve Klinik Mikrobiyoloji. Ankara Güneş Kitabevi Ltd. Şti. 1999; 571-577.

2. Pappas G, Papadimitriou P, Akritidis N, Christou L, Tsianos EV. The new global map of human brucellosis. Lancet Infect Dis 2006; 6: 91-99.

3. Black TF. Brucellosis. In: Cohen J, Powderly WG, editors. Infectious diseases. 2nd ed., St. Louis: Mosby; 2004; 1665-1667.

4. Doganay M, Alp ME. Bruselloz. Eds: Wilke Topcu, Soyletir G, Doganay M Enfeksiyon hastalıkları ve mikrobiyoloji. Nobel Tip Kitabevi. Cilt 1: Bask1 3; 2008; 897-909.

5. Yuce A, Cavus SA. Turkiye'de bruselloz: genel bakıs. Klimik derg 2006; 19: 87-97.

6. Young EJ. Brucella species. In: Mandell GL, Bennett JE, Dolin R, editors. Principles and practice of infectious diseases. 6th ed., Philadelphia: Churchill Livingstone; 2005; 2669_ 72.

7. Gotuzzo E, Celillo E, Brucella. In: Gorbach SI, Bartlett JG, Blacklow NR, editors. Infectious diseases. Philadelphia: Harcourt Brace Jovanovich Inc; 1992; 1513-1518.

8. Hall WH. Brucellosis. In: Evans AS, Brachman PS, editors. Bacterial infections of humans. 2nd ed., New York: Plenum Publishing Corp; 1991; 133-151.

9. Pittman QJ. A neuro-endocrine-immune symphony. J Neuroendocrinol 2011; 23: 12961297.

10. Petrovsky N. Towards a unified model of neuroendocrine-immune interaction. Immunol Cell Biol 2001; 79: 350-357.

11. Polyzos SA, Anastasilakis AD, Efstathiadou ZA, et al. Irisin in metabolic disease. Endocrine 2018; 59: 260-274.

12. Atici E, Mogulkoc R, Baltaci AK, Menevse E. Both hypothyroidism and hyperthyroidism increase plasma irisin levels in rats. Horm Mol Biol Clin Investig 2017; 33: 54.

13. Bostrom P, Wu J, Jedrychowski MP, et al. A PGC1- $\alpha$-dependent myokine that drives brown-fat-like development

of white fat and thermogenesis. Nature 2012; 481: 463-468.

14. Roca-Rivada A, Castelao C, Senin LL, et al. FNDC5/irisin is not only a myokine but also an adipokine. Plos One 2013; 8: 60563.

15. Ugras S, Ozcelik O. Impact of exercise induced skeletal muscle strain on energy regulatory hormones of irisin and nesfatin-1 in sedentary males. Turkish Bulletin of Hygiene and Experimental Biology 2020; 77: 227-232.

16. Ozcelik O, Algul S, Yilmaz B. Nesfatin-1 and irisin levels in response to the soccer matches performed in morning, afternoon, and at night in young trained male subjects. Cell Moll Biol. 2018; 64: $130-133$

17. Algul S, Ozdenk C, Ozcelik O. Variations in leptin, nesfatin-1 and irisin levels induced by aerobic exercise in young trained and untrained male subjects.Biol Sport 2017; 34: 339-344.

18. Olt S, Ergenc H, Acikgoz SB. Predictive contribution of neutrophil/lymphocyte ratio in diagnosis of brucellosis. Biomed Res Int 2015; 2015: 210502

19. Demir F, Karadeniz C, Ozdemir R, et al. Usefulness of neutrophil to lymphocyte ratio in prediction of coronary artery lesions in patients with Kawasaki disease. Balkan Med J 2015; 32: 371-376.

20. Uygur F, Tanriverdi H, Aktop $\mathrm{Z}$, et al. The neutro- phil-to-lymphocyte ratio in patients with obstructive sleep apnoea syndrome and its relationship with cardiovascular disease. Heart Lung 2016; 45: 121-125.

21. Kucuk A, Erol MF, Senel S, et al. The role of neutrophil lymphocyte ratio to leverage the differential diagnosis of familial Mediterranean fever attack and acute appendicitis. Korean J Intern Med 2016; 31: 386-391.

22. Atan D, Ikinciogullari A, Koseoglu S, et al. New predictive parameters of Bell's palsy: neutrophil to lymphocyte ratio and platelet to lymphocyte ratio. Balkan Med J 2015; 32: 167-170.

23. Yin X, Xiao Y, Li F, Qi S, Yin Z, Gao J. Prognostic role of neutrophil-to-lymphocyte ratio in prostate cancer: a sys- tematic review and metaanalysis. Medicine (Baltimore) 2016; 95: 2544.

24. Alvarado A. A practical score for the early diagnosis of acute appendicitis. Ann Emerg Med 1986; 15: 557-564.

25. Sarac F, Buyukbese Sarsu S, Yeniocak S, et al. The Diagnostic Value of Irisin in Pediatric Patients with Acute Abdominal Pain. Emerg Med Int 2018; 2018: 3296535.

26. Gouveia MC, Vella JP, Cafeo FR, Affonso Fonseca FL, Bacci MR. Association between irisin and major chronic diseases: a review. Eur Rev Med Pharmacol Sci 2016; 20: 4072-4077.

27. Bashar SM, Samir El-Sherbeiny SM, Boraie MZ. Correlation between the blood level of irisin and the severity of acute myocardial infarction in exercise-trained rats $\mathrm{J}$ Basic Clin Physiol PharmacoL 2018; 30: 59-71. 